\title{
Generating Natural Motion in an Android by Mapping Human Motion
}

\author{
Daisuke Matsui ${ }^{1}$, Takashi Minato ${ }^{1}$, Karl F. MacDorman ${ }^{2}$ \\ and Hiroshi Ishiguro ${ }^{1}$ \\ ${ }^{1}$ Osaka University, ${ }^{2}$ Indiana University \\ ${ }^{1}$ Japan, ${ }^{2}$ USA
}

\section{Introduction}

Much effort in recent years has focused on the development of such mechanical-looking humanoid robots as Honda's Asimo and Sony's Qrio with the goal of partnering them with people in daily situations. Just as an industrial robot's purpose determines its appearance, a partner robot's purpose will also determine its appearance. Partner robots generally adopt a roughly humanoid appearance to facilitate communication with people, because natural interaction is the only task that requires a humanlike appearance. In other words, humanoid robots mainly have significance insofar as they can interact naturally with people. Therefore, it is necessary to discover the principles underlying natural interaction to establish a methodology for designing interactive humanoid robots.

Kanda et al. (Kanda et al., 2002) have tackled this problem by evaluating how the behavior of the humanoid robot "Robovie" affects human-robot interaction. But Robovie's machinelike appearance distorts our interpretation of its behavior because of the way the complex

$\varepsilon$ relationship between appearance and behavior influences the interaction. Most research on $\underset{\odot}{0}$ interactive robots has not evaluated the effect of appearance (for exceptions, see (Goetz et $\stackrel{\Phi}{.}$ al., 2003; DiSalvo et al., 2002)) - and especially not in a robot that closely resembles a o person. Thus, it is not yet clear whether the most comfortable and effective human-robot O communication would come from a robot that looks mechanical or human. However, we $\underset{.1}{.1}$ may infer a humanlike appearance is important from the fact that human beings have developed neural centers specialized for the detection and interpretation of hands and faces (Grill-Spector et al., 2004; Farah et al., 2000; Carmel \& Bentin, 2002). A robot that closely (1) resembles humans in both looks and behavior may prove to be the ultimate communication Of device insofar as it can interact with humans the most naturally. ${ }^{1}$ We refer to such a device ฮิ as an android to distinguish it from mechanical-looking humanoid robots. When we $\widetilde{\pi}$ investigate the essence of how we recognize human beings as human, it will become clearer s how to produce natural interaction. Our study tackles the appearance and behavior problem \& with the objective of realizing an android and having it be accepted as a human being (Minato et al., 2006).

${ }^{1}$ We use the term natural to denote communication that flows without seeming stilted, forced, bizarre, or inhuman. 
Ideally, to generate humanlike movement, an android's kinematics should be functionally equivalent to the human musculoskeletal system. Some researchers have developed a joint system that simulates shoulder movement (Okada et al., 2002) and a muscle-tendon system to generate humanlike movement (Yoshikai et al., 2003). However, these systems are too bulky to be embedded in an android without compromising its humanlike appearance. Given current technology, we embed as many actuators as possible to provide many degrees of freedom insofar as this does not interfere with making the android look as human as possible (Minato et al., 2006). Under these constraints, the main issue concerns how to move the android in a natural way so that its movement may be perceived as human.

A straightforward way to make a robot's movement more humanlike is to imitate human motion. Kashima and Isurugi (Kashima \& Isurugi, 1998) extracted essential properties of human arm trajectories and designed an evaluation function to generate robot arm trajectories accordingly. Another method is to copy human motion as measured by a motion capture system to a humanoid robot. Riley et al. (Riley et al., 2000) and Nakaoka et al. (Nakaoka et al., 2003) calculated a subject's joint trajectories from the measured positions of markers attached to the body and fed them to the joints of a humanoid robot. In these studies the authors assumed the kinematics of the robot to be similar to that of a human body. However, since the actual kinematics and joint structures are different between human and robot bodies, calculating the joint angles from only the human motion data could in some cases result in visibly different motion. This is especially a risk for androids because their humanlike form makes us more sensitive to deviations from human ways of moving. Thus, slight differences could strongly influence whether the android's movement is perceived as natural or human. Furthermore, these studies did not evaluate the naturalness of robot motions.

Hale et al. (Hale et al., 2003) proposed several evaluation functions to generate a joint trajectory (e.g., minimization of jerk) and evaluated the naturalness of generated humanoid robot movements according to how human subjects rated their naturalness. In the computer animation domain, researchers have tackled a motion synthesis with motion capture data (e.g., (Gleicher, 1998)). However, we cannot apply their results directly; we must instead repeat their experiment with an android because the results from an android testbed could be quite different from those of a humanoid testbed. For example, Mori described a phenomenon he termed the "uncanny valley" (Mori, 1970; Fong et al., 2003), which relates to the relationship between how humanlike a robot appears and a subject's perception of familiarity. According to Mori, a robot's familiarity increases with its similarity until a certain point is reached at which slight "nonhuman" imperfections cause the robot to appear repulsive (Fig. 1). This would be an issue if the similarity of androids fell into the chasm. (Mori believes mechanical-looking humanoid robots lie on the left of the first peak.) This nonmonotonic relationship can distort the evaluation proposed in existing studies. Therefore, it is necessary to develop a motion generation method in which the generated "android motion" is perceived as human.

This paper proposes a method to transfer human motion measured by a motion capture system to the android by copying changes in the positions of body surfaces. This method is called for because the android's appearance demands movements that look human, but its kinematics is sufficiently different that copying joint-angle information would not yield good results. Comparing the similarity of the android's visible movement to that of a human being enables us to develop more natural movements for the android. 


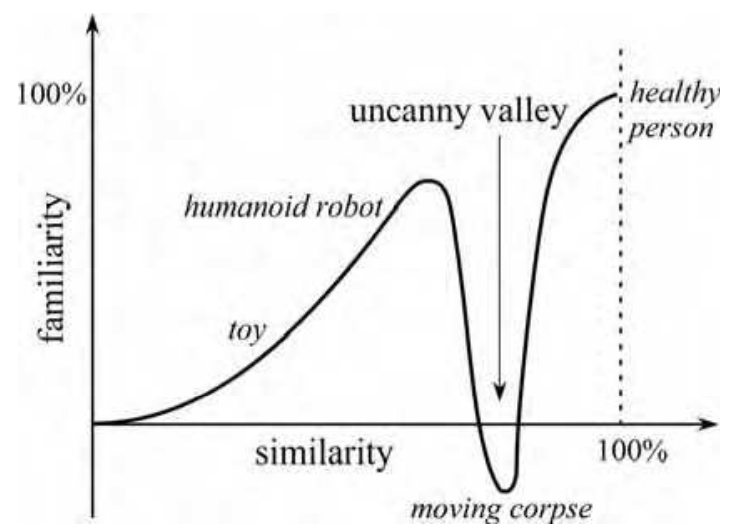

Figure 1. Uncanny valley (Mori, 1970; Fong et al., 2003)

In the following sections, we describe the developed android and mention the problem of motion transfer and our basic idea about the way to solve it. Then we describe the proposed method in detail and show experimental results from applying it to the android.

\section{The Developed Android}

Fig. 2 shows the developed android called Repliee Q2. The android is modeled after a Japanese woman. The standing height is about $160 \mathrm{~cm}$. The skin is composed of a kind of silicone that feels like human skin. The silicone skin covers the neck, head, and forearms, with clothing covering other body parts. Unlike Repliee R1 (Minato et al., 2004), the silicone skin does not cover the entire body so as to facilitate flexibility and a maximal range of motion. Forty-two highly sensitive tactile sensors composed of PVDF film are mounted under the android's skin and clothes over the entire body, except for the shins, calves, and feet. Since the output value of each sensor corresponds to its deforming rate, the sensors can distinguish different kinds of touch ranging from stroking to hitting. The soft skin and tactile sensors give the android a human appearance and enable natural tactile interaction. The android is driven by air actuators (air cylinders and air motors) that give it 42 degrees of freedom (DoFs) from the waist up. The legs and feet are not powered; it can neither stand up nor move from a chair. A high power-to-weight ratio is necessary for the air actuator in order to mount multiple actuators in the human-sized body.

The configuration of the DoFs is shown in Table 1. Fig. 4 shows the kinematic structure of the body, excluding the face and fingers. Some joints are driven by the air motors and others adopt a slider-crank mechanism. The DoFs of the shoulders enable them to move up and down and backwards and forwards; this shoulder structure is more complicated than that of most existing humanoid robots. Moreover, parallel link mechanisms adopted in some parts complicate the kinematics of the android, for example in the waist. The android can generate a wide range of motions and gestures as well as various kinds of micro-motions such as the shoulder movements typically caused by human breathing. Furthermore, the android can make some facial expressions and mouth shapes, as shown in Fig. 3. Because the android has servo controllers, it can be controlled by sending data on the desired joint angles (cylinder positions and rotor angles) from a host computer. The compliance of the air actuator makes for safer interaction, with movements that are generally smoother than other 
systems typically used. Because of the complicated dynamics of the air actuator, executing the trajectory tracking control is difficult.

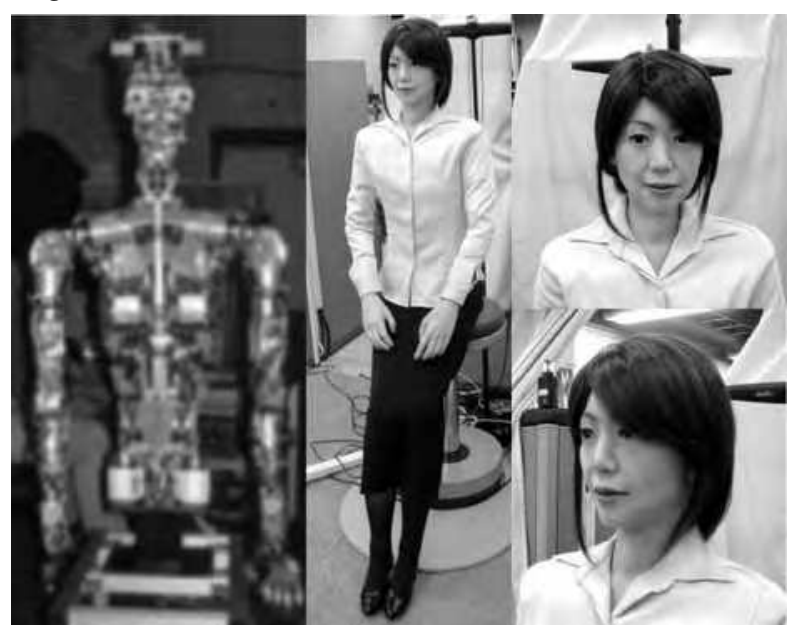

Figure 2. The developed android "Repliee Q2"

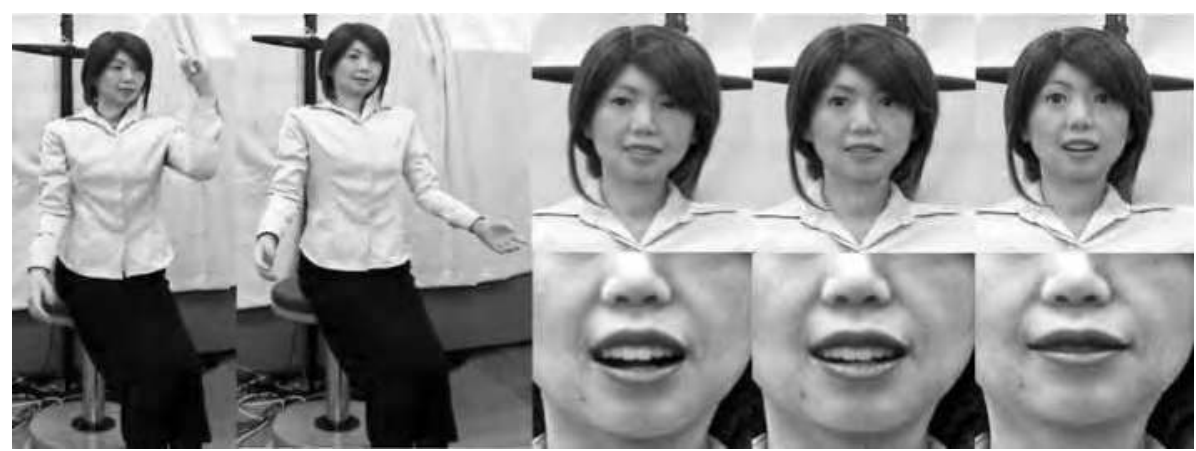

Figure 3. Examples of motion and facial expressions

\begin{tabular}{|c|c|}
\hline \multicolumn{2}{|c|}{ Degree of freedom } \\
\hline Eyes & pan $\times 2+$ tilt $\times 1$ \\
\hline Face & eyebrows $\times 1+$ eyelids $\times 1+$ cheeks $\times 1$ \\
\hline Mouth & 7 (including the upper and lower lips) \\
\hline Neck & 3 \\
\hline Shoulder & $5 \times 2$ \\
\hline Elbow & $2 \times 2$ \\
\hline Wrist & $2 \times 2$ \\
\hline Fingers & $2 \times 2$ \\
\hline Torso & 4 \\
\hline
\end{tabular}

Table 1. The DoF configuration of Repliee Q2. 


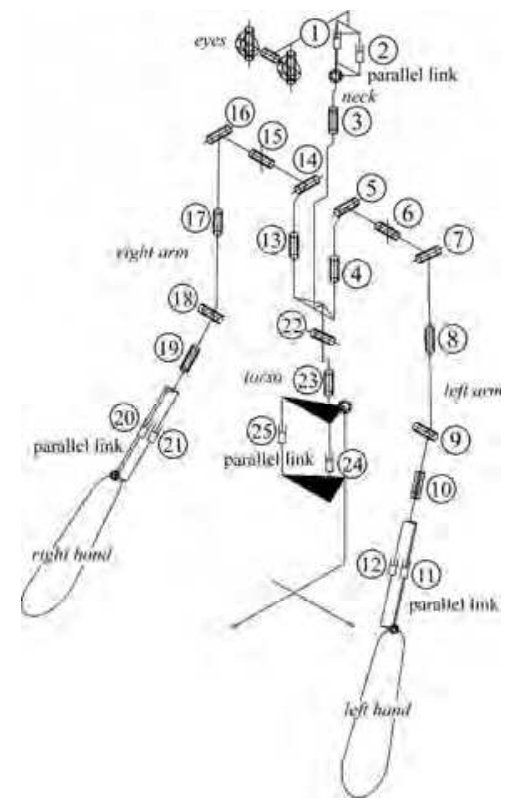

Figure 4. Kinematic structure of the android

\section{Transferring Human Motion}

\subsection{The basic idea}

One method to realize humanlike motion in a humanoid robot is through imitation. Thus, we consider how to map human motion to the android. Most previous research assumes the kinematics of the human body is similar to that of the robot except for the scale. Thus, they aim to reproduce human motion by reproducing kinematic relations across time and, in particular, joint angles between links. For example, the three-dimensional locations of markers attached to the skin are measured by a motion capture system, the angles of the body's joints are calculated from these positions, and these angles are transferred to the joints of the humanoid robot. It is assumed that by using a joint angle space (which does not represent link lengths), morphological differences between the human subject and the humanoid robot can be ignored.

However, there is potential for error in calculating a joint angle from motion capture data. The joint positions are assumed to be the same between a humanoid robot and the human subject who serves as a model; however, the kinematics in fact differs. For example, the kinematics of Repliee Q2's shoulder differs significantly from those of human beings. Moreover, as human joints rotate, each joint's center of rotation changes, but joint-based approaches generally assume this is not so. These errors are perhaps more pronounced in Repliee Q2, because the android has many degrees of freedom and the shoulder has a more complex kinematics than existing humanoid robots. These errors are more problematic for an android than a mechanical-looking humanoid robot because we expect natural human motion from something that looks human and are disturbed when the motion instead looks inhuman. 
To create movement that appears human, we focus on reproducing positional changes at the body's surface rather than changes in the joint angles. We then measure the postures of a person and the android using a motion capture system and find the control input to the android so that the postures of person and android become similar to each other.

\subsection{The method to transfer human motion}

We use a motion capture system to measure the postures of a human subject and the android. This system can measure the three-dimensional positions of markers attached to the surface of bodies in a global coordinate space. First, some markers are attached to the android so that all joint motions can be estimated. The reason for this will become clear later. Then the same numbers of markers are attached to corresponding positions on the subject's body. We must assume the android's surface morphology is not too different from the subject's.

We use a three-layer neural network to construct a mapping from the subject's posture $x_{h}$ to the android's control input $\boldsymbol{q}_{a}$, which is the desired joint angle. The reason for the network is that it is difficult to obtain the mapping analytically. To train a neural network to map from $x_{h}$ to $\boldsymbol{q}_{a}$ would require thousands of pairs of $\boldsymbol{x}_{h}, \boldsymbol{q}_{a}$ as training data, and the subject would need to assume the posture of the android for each pair. We avoid this prohibitively lengthy task in data collection by adopting feedback error learning (FEL) to train the neural network. Kawato et al. (Kawato et al., 1987) proposed feedback error learning as a principle for learning motor control in the brain. This employs an approximate way of mapping sensory errors to motor errors that subsequently can be used to train a neural network (or other method) by supervised learning. Feedback-error learning neither prescribes the type of neural network employed in the control system nor the exact layout of the control circuitry. We use it to estimate the error between the postures of the subject and the android and feed the error back to the network.

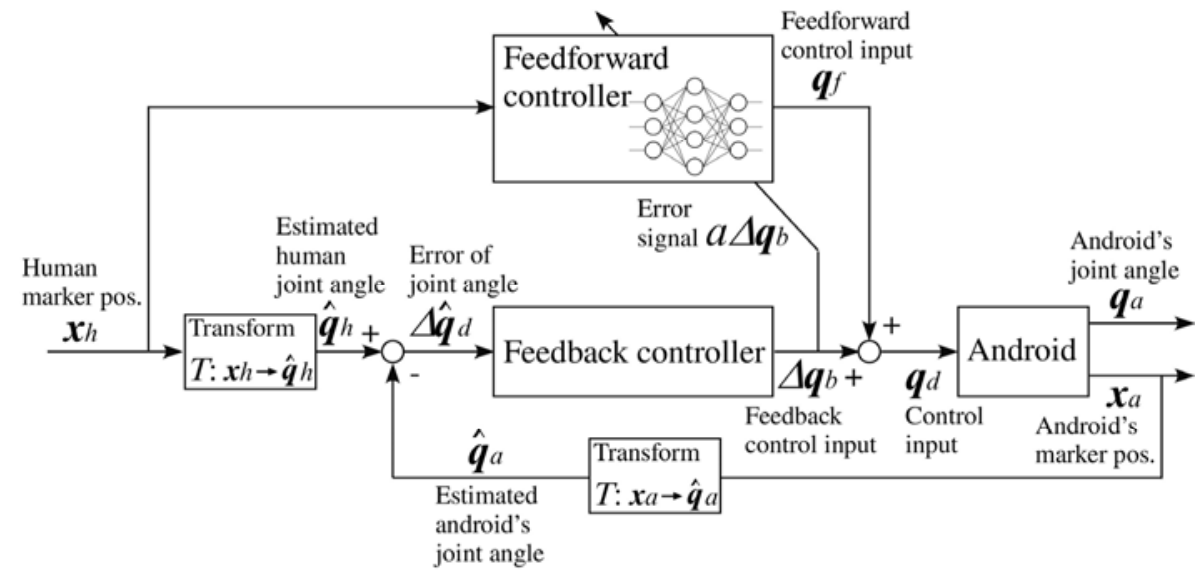

Figure 5. The android control system

Fig. 5 shows the block diagram of the control system, where the network mapping is shown as the feedforward controller. The weights of the feedforward neural network are learned by means of a feedback controller. The method has a two-degrees-of-freedom control 
architecture. The network tunes the feedforward controller to be the inverse model of the plant. Thus, the feedback error signal is employed as a teaching signal for learning the inverse model. If the inverse model is learned exactly, the output of the plant tracks the reference signal by feedforward control. The subject and android's marker positions are represented in their local coordinates $\boldsymbol{x}_{h}, \boldsymbol{x}_{a} \in \boldsymbol{R}^{3 m}$; the android's joint angles $\boldsymbol{q}_{a} \in \boldsymbol{R}^{n}$ can be observed by a motion capture system and a potentiometer, where $m$ is the number of markers and $n$ is the number of DoFs of the android.



(a) Feedback of maker position error

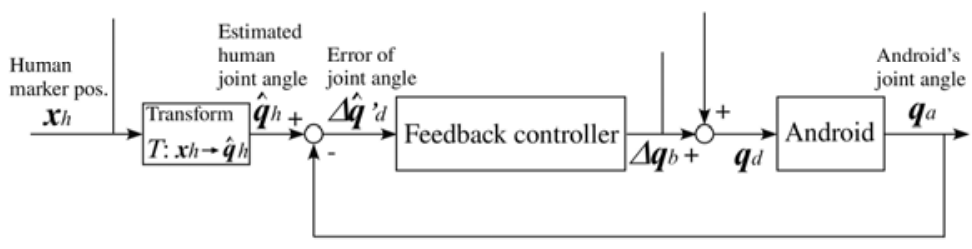

(b) Error estimation with the android's joint angle measured by the potentiometer

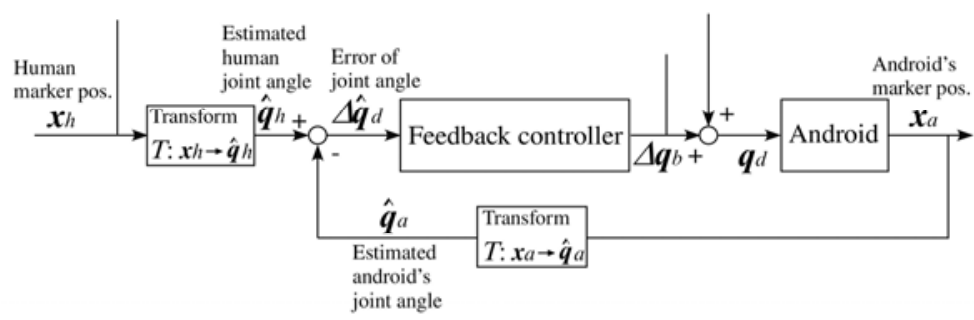

(c) Error estimation with the android's joint angle estimated from the android's marker position

Fig 6. The feedback controller with and without the estimation of the android's joint angle

The feedback controller is required to output the feedback control input $\Delta \boldsymbol{q}_{b}$ so that the error in the marker's position $\Delta x_{d}=x_{a}-x_{h}$ converges to zero (Fig. 6(a)). However, it is difficult to obtain $\Delta \boldsymbol{q}_{b}$ from $\Delta \boldsymbol{x}_{d}$. To overcome this, we assume the subject has roughly the same kinematics as the android and obtain the estimated joint angle $\hat{\boldsymbol{q}}_{h}$ simply by calculating the Euler angles (hereafter the transformation from marker positions to joint angles is described as $T){ }^{2}$ Converging $\boldsymbol{q}_{a}$ to $\hat{\boldsymbol{q}}_{h}$ does not always produce identical postures because $\hat{\boldsymbol{q}}_{h}$ is an

2 There are alternatives to using the Euler angles such as angle decomposition (Grood \& Suntay, 1983), which has the advantage of providing a sequence independent representation, or least squares, to calculate the helical axis and rotational angle (Challis, 1995; Veldpaus et al., 1988). This last method provides higher accuracy when many markers are used but has an increased risk of marker crossover. 
approximate joint angle that may include transformation error (Fig. 6(b)). Then we obtain the estimated joint angle of the android $\hat{\boldsymbol{q}}_{a}$ using the same transformation $T$ and the feedback control input to converge $\hat{\boldsymbol{q}}_{a}$ to $\hat{\boldsymbol{q}}_{h}$ (Fig. 6(c)). This technique enables $\boldsymbol{x}_{a}$ to approach $x_{h}$. The feedback control input approaches zero as learning progresses, while the neural network constructs the mapping from $\boldsymbol{x}_{h}$ to the control input $\boldsymbol{q}_{d}$. We can evaluate the apparent posture by measuring the android posture.

In this system we could have made another neural network for the mapping from $x_{a}$ to $\boldsymbol{q}_{a}$ using only the android. As long as the android's body surfaces are reasonably close to the subject's, we can use the mapping to make the control input from $x_{h}$. Ideally, the mapping must learn every possible posture, but this is quite difficult. Therefore, it is still necessary for the system to evaluate the error in the apparent posture.

\section{Experiment to Transfer Human Motion}

\subsection{Experimental setting}

To verify the proposed method, we conducted an experiment to transfer human motion to the android Repliee Q2. We used 21 of the android's 42 DoFs $(n=21)$ by excluding the 13 DoFs of the face, the 4 of the wrists (the cylinders 11, 12, 20, and 21 in Fig. 4), and the 4 of the fingers. We used a Hawk Digital System, ${ }^{3}$ which can track more than 50 markers in realtime. The system is highly accurate with a measurement error of less than $1 \mathrm{~mm}$. Twenty markers were attached to the subject and another 20 to the android as shown in Fig. 7 ( $m=$ 20). Because the android's waist is fixed, the markers on the waist set the frame of reference for an android-centered coordinate space. To facilitate learning, we introduce a representation of the marker position $x_{h}, x_{a}$ as shown in Fig. 8. The effect of waist motions is removed with respect to the markers on the head. To avoid accumulating the position errors at the end of the arms, vectors connecting neighboring pairs of markers represent the positions of the markers on the arms. We used arc tangents for the transformation $T$, in which the joint angle is an angle between two neighboring links where a link consists of a straight line between two markers.

The feedback controller outputs $\Delta \boldsymbol{q}_{b}=K \Delta \hat{\boldsymbol{q}}_{d}$, where the gain $K$ consists of a diagonal matrix. There are 60 nodes in the input layer (20 markers $\times x, y, z), 300$ in the hidden layer, and 21 in the output layer (for the $21 \mathrm{DoFs}$ ). Using 300 units in the hidden layer provided a good balance between computational efficiency and accuracy. Using significantly fewer units resulted in too much error, while using significantly more units provided only marginally higher accuracy but at the cost of slower convergence. The error signal to the network is $t=$ $a \Delta \boldsymbol{q}_{b}$, where the gain $a$ is a small number. The sampling time for capturing the marker positions and controlling the android is $60 \mathrm{~ms}$. Another neural network which has the same structure previously learned the mapping from $x_{a}$ to $\boldsymbol{q}_{a}$ to set the initial values of the weights. We obtained 50,000 samples of training data $\left(\boldsymbol{x}_{a}\right.$ and $\left.\boldsymbol{q}_{a}\right)$ by moving the android randomly. The learned network is used to set the initial weights of the feedforward network.

\footnotetext{
${ }^{3}$ Motion Analysis Corporation, Santa Rosa, California. http://www.motionanalysis.com/
} 


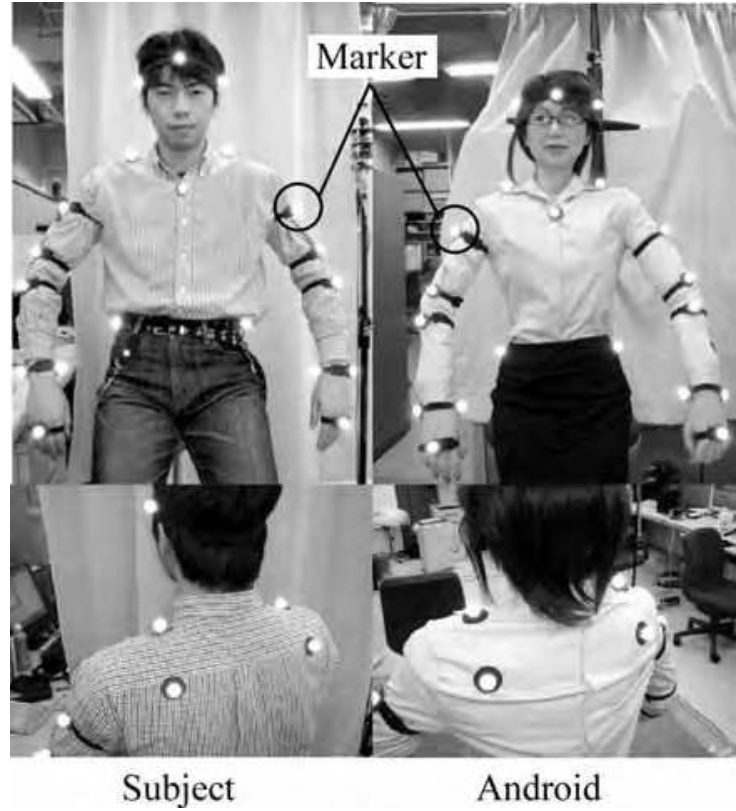

Figure 7. The marker positions corresponding to each other
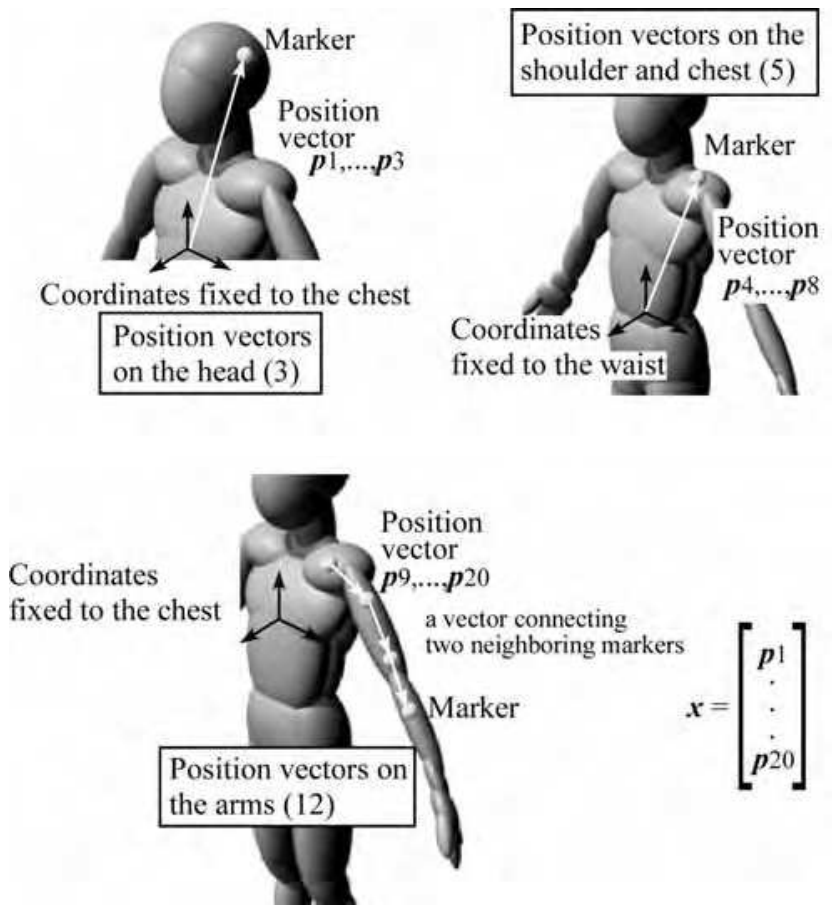

Figure 8 . The representation of the marker positions. A marker's diameter is about $18 \mathrm{~mm}$ 


\subsection{Experimental results and analysis}

\subsubsection{Surface similarity between the android and subject}

The proposed method assumes a surface similarity between the android and the subject. However, the male subject whom the android imitates in the experiments was $15 \mathrm{~cm}$ taller than the women after whom the android was modeled. To check the similarity, we measured the average distance between corresponding pairs of markers when the android and subject make each of the given postures; the value was $31 \mathrm{~mm}$ (see the Fig. 7). The gap is small compared to the size of their bodies, but it is not small enough.

\subsubsection{The learning of the feedforward network}

To show the effect of the feedforward controller, we plot the feedback control input averaged among the joints while learning from the initial weights in Fig. 9. The abscissa denotes the time step (the sampling time is $60 \mathrm{~ms}$.) Although the value of the ordinate does not have a direct physical interpretation, it corresponds to a particular joint angle. The subject exhibited various fixed postures. When the subject started to make the posture at step 0 , error increased rapidly because network learning had not yet converged. The control input decreases as learning progresses. This shows that the feedforward controller learned so that the feedback control input converges to zero.

Fig. 10 shows the average position error of a pair of corresponding markers. The subject also gave an arbitrary fixed posture. The position errors and the feedback control input both decreased as the learning of the feedforward network converged. The result shows the feedforward network learned the mapping from the subject's posture to the android control input, which allows the android to adopt the same posture. The android's posture could not match the subject's posture when the weights of the feedforward network were left at their initial values. This is because the initial network was not given every possible posture in the pre-learning phase. The result shows the effectiveness of the method to evaluate the apparent posture.

\subsubsection{Performance of the system at following fast movements}

To investigate the performance of the system, we obtained a step response using the feedforward network after it had learned enough. The subject put his right hand on his knee and quickly raised the hand right above his head. Fig. 11 shows the height of the fingers of the subject and android. The subject started to move at step 5 and reached the final position at step 9, approximately 0.24 seconds later. In this case the delay is 26 steps or 1.56 seconds. The arm moved at roughly the maximum speed permitted by the hardware. The android arm cannot quite reach the subject's position because the subject's position was outside of the android's range of motion. Clearly, the speed of the subject's movement exceeds the android's capabilities. This experiment is an extreme case. For less extreme gestures, the delay will be much less. For example, for the sequence in Fig. 12, the delay was on average seven steps or 0.42 seconds. 


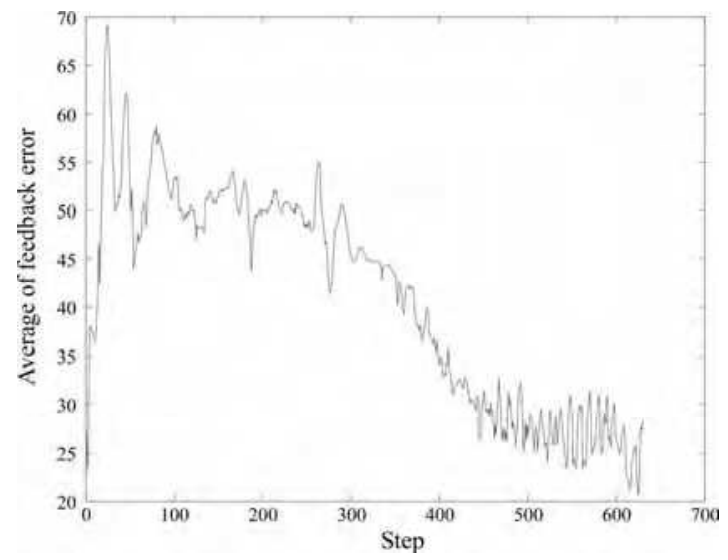

Figure 9. The change of the feedback control input with learning of the network

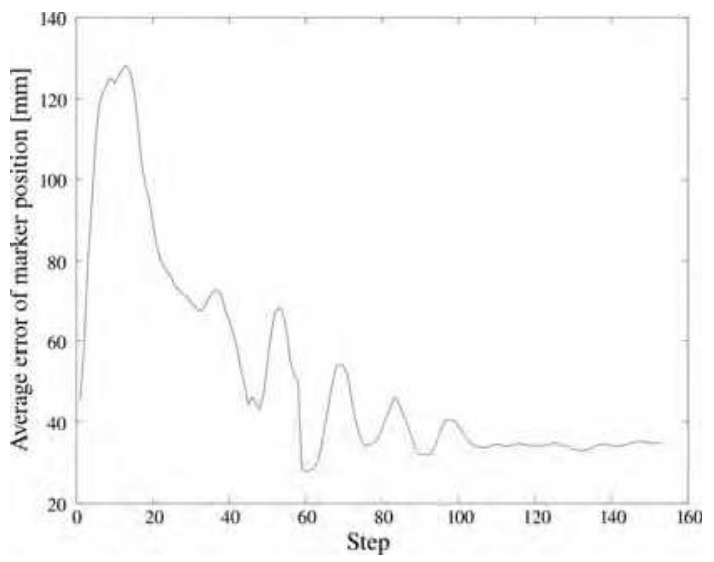

Figure 10. The change of the position error with learning of the network

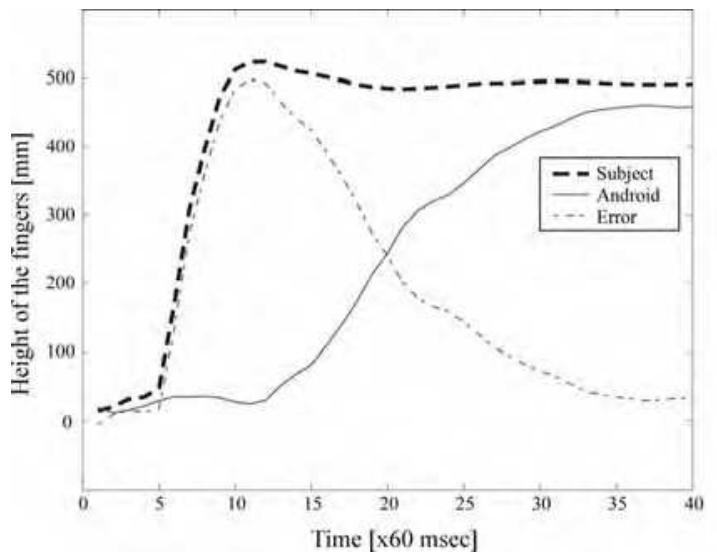

Figure 11. The step response of the android 

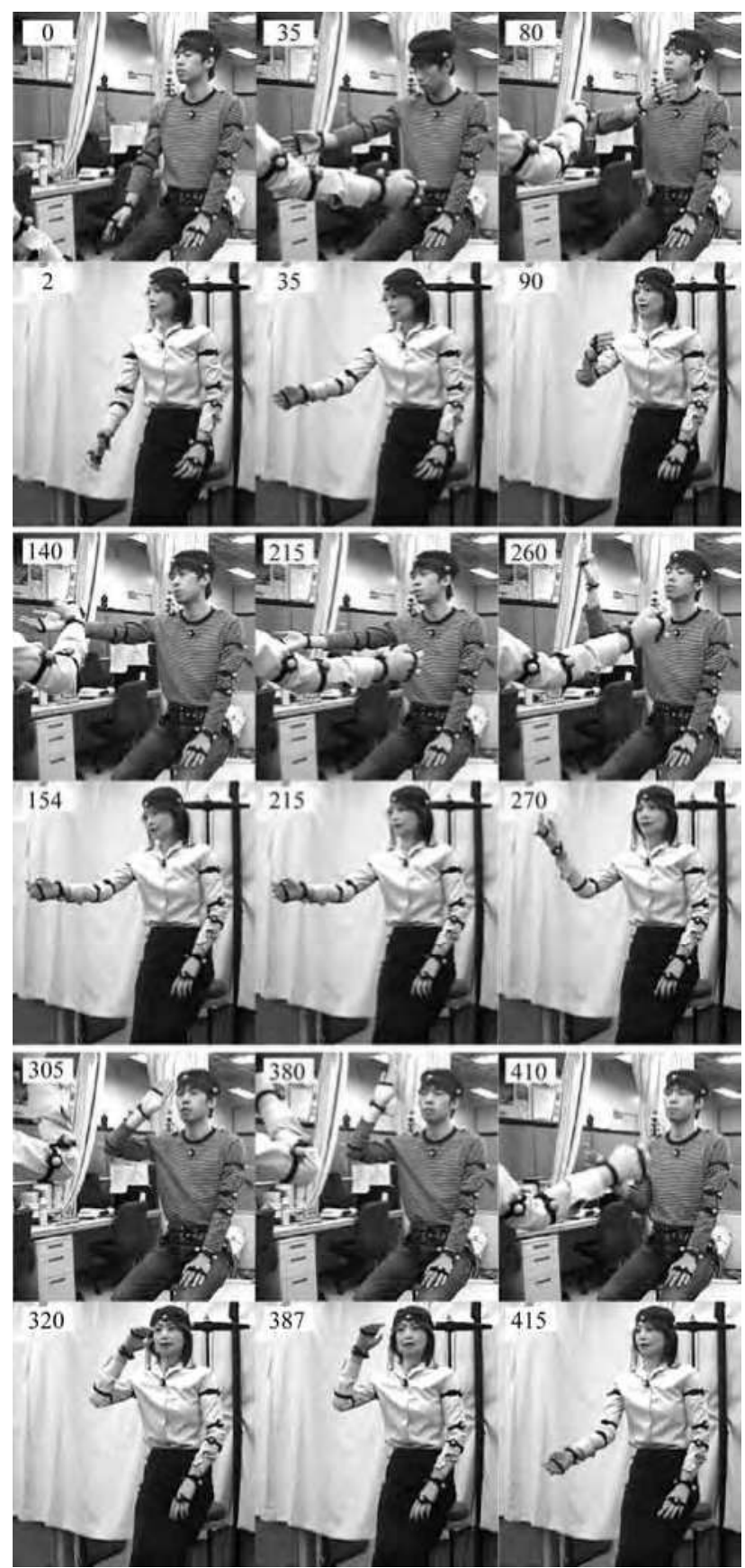

Figure 12. The generated android's motion compared to the subject's motion. The number represents the step. 


\subsubsection{The generated android motion}

Fig. 12 shows the subject's postures during a movement and the corresponding postures of the android. The value denotes the time step. The android followed the subject's movement with some delay (the maximum is 15 steps, that is, 0.9 seconds). The trajectories of the positions of the android's markers are considered to be similar to those of the subject, but errors still remain, and they cannot be ignored. While we can recognize that the android is making the same gesture as the subject, the quality of the movement is not the same. There are a couple of major causes of this:

- The kinematics of the android is too complicated to represent with an ordinary neural network. To avoid this limitation, it is possible to introduce the constraint of the body's branching in the network connections. Another idea is to introduce a hierarchical representation of the mapping. A human motion can be decomposed into a dominant motion that is at least partly driven consciously and secondary motions that are mainly nonconscious (e.g., contingent movements to maintain balance, such autonomic responses as breathing). We are trying to construct a hierarchical representation of motion not only to reduce the computational complexity of learning but to make the movement appear more natural.

- The method deals with a motion as a sequence of postures; it does not precisely reproduce higher order properties of motion such as velocity and acceleration because varying delays can occur between the subject's movement and the android's imitation of it. If the subject moves very quickly, the apparent motion of the android differs. Moreover, a lack of higher order properties prevents the system from adequately compensating for the dynamic characteristics of the android and the delay of the feedforward network.

- The proposed method is limited by the speed of motion. It is necessary to consider the properties to overcome the restriction, although the android has absolute physical limitations such as a fixed compliance and a maximum speed that is less than that of a typical human being.

Although physical limitations cannot be overcome by any control method, there are ways of finessing them to ensure movements still look natural. For example, although the android lacks the opponent musculature of human beings, which affords a variable compliance of the joints, the wobbly appearance of such movements as rapid waving, which are high in both speed and frequency, can be overcome by slowing the movement and removing repeated closed curves in the joint angle space to eliminate lag caused by the slowed movement. If the goal is humanlike movement, one approach may be to query a database of movements that are known to be humanlike to find the one most similar to the movement made by the subject, although this begs the question of where those movements came from in the first place. Another method is to establish criteria for evaluating the naturalness of a movement (Kashima \& Isurugi, 1998). This is an area for future study.

\subsection{Required improvement and future work}

In this paper we focus on reproducing positional changes at the body's surface rather than changes in the joint angles to generate the android's movement. Fig. 6(a) is a straightforward method to implement the idea. This paper has adopted the transformation $T$ from marker positions to estimated joint angles because it is difficult to derive a feedback controller which produces the control input $\Delta \boldsymbol{q}_{b}$ only from the error in the marker's 
positional error $\Delta x_{d}$ analytically. We actually do not know which joints should be moved to remove a positional error at the body's surface. This relation must be learned, however, the transformation $T$ could disturb the learing. Hence, it is not generally guaranteed that the feedback controller which converges the estimated joint angle $\hat{\boldsymbol{q}}_{a}$ to $\hat{\boldsymbol{q}}_{h}$ enables the marker's position $x_{a}$ to approach $x_{h}$. The assumption that the android's body surfaces are reasonably close to the subject's could avoid this problem, but the feedback controller shown in Fig. 6(a) is essentially necessary for mapping the apparent motion. It is possible to find out how the joint changes relate to the movements of body surfaces by analyzing the weights of the neural network of the feedforward controller. A feedback controller could be designed to output the control input based on the error in the marker's position with the analyzed relation. Concerning the design of the feedback controller, Oyama et al. (Oyama et al., 2001a; Oyama et al., 2001b; Oyama et al., 2001c) proposed several methods for learning both of feedback and feedforward controllers using neural networks. This is one potential method to obtain the feedback controller shown in Fig. 6(a). Assessment of and compensation for deformation and displacement of the human skin, which cause marker movement with respect to the underlying bone (Leardini et al., 2005), are also useful in designing the feedback controller.

We have not dealt with the android's gaze and facial expressions in the experiment; however, if gaze and facial expressions are unrelated to hand gestures and body movements, the appearance is often unnatural, as we have found in our experiments. Therefore, to make the android's movement appear more natural, we have to consider a method to implement the android's eye movements and facial expressions.

\section{Conclusion}

This paper has proposed a method of implementing humanlike motions by mapping their three-dimensional appearance to the android using a motion capture system. By measuring the android's posture and comparing it to the posture of a human subject, we propose a new method to evaluate motion sequences along bodily surfaces. Unlike other approaches that focus on reducing joint angle errors, we consider how to evaluate differences in the android's apparent motion, that is, motion at its visible surfaces. The experimental results show the effectiveness of the evaluation: the method can transfer human motion. However, the method is restricted by the speed of the motion. We have to introduce a method to deal with the dynamic characteristics (Ben=Amor et al., 2007) and physical limitations of the android. We also have to evaluate the method with different subjects. We would expect to generate the most natural and accurate movements using a female subject who is about the same height as the original woman on which the android is based. Moreover, we have to evaluate the human likeness of the visible motions by the subjective impressions the android gives experimental subjects and the responses it elicits, such as eye contact (Minato et al., 2006; Shimada et al., 2006), autonomic responses, and so on. Research in these areas is in progress.

\section{Acknowledgment}

We developed the android in collaboration with Kokoro Company, Ltd 


\section{References}

Ben=Amor, H., Ikemoto, S., Minato, T., Jung, B., and Ishiguro, H. (2007). A neural framework for robot motor learning based on memory consolidation. Proceedings of International Conference on Adaptive and Natural Computing Algorithms, Warsaw, Poland, 2007.04.

Carmel, D. and Bentin, S. (2002). Domain specificity versus expertise: Factors influencing distinct processing of faces. Cognition, Vol. 83, (1-29), ISSN:0010-0277.

Challis, J. H. (1995). A procedure for determining rigid body transformation parameters. Journal of Biomechanics, Vol. 28, (733-737), ISSN:0021-9290.

DiSalvo, C. F., Gemperle, F., Forlizzi, J., and Kiesler, S. (2002). All robots are not created equal: The design and perception of humanoid robot heads. Proceedings of the Symposium on Designing Interactive Systems, pp. 321-326, ISBN:1-58113-515-7, London, England, 2002.06.

Farah, M. J., Rabinowitz, C., Quinn, G. E., and Liu, G. T. (2000). Early commitment of neural substrates for face recognition. Cognitive Neuropsychology, Vol. 17, (117-123), ISSN:0264-3294.

Fong, T., Nourbakhsh, I., and Dautenhahn, K. (2003). A survey of socially interactive robots. Robotics and Autonomous Systems, Vol. 42, (143-166), ISSN:0921-8890.

Gleicher, M. (1998). Retargetting motion to new characters. Proceedings of the International Conference on Computer Graphics and Interactive Techniques, pp. 33-42, ISBN:0-89791999-8, Florida, USA, 2003.07.

Goetz, J., Kiesler, S., and Powers, A. (2003). Matching robot appearance and behavior to tasks to improve human-robot cooperation. Proceedings of the Workshop on Robot and Human Interactive Communication, pp. 55-60, ISBN:0-7803-8136-X, California, USA, 2003.10.

Grill-Spector, K., Knouf, N., and Kanwisher, N. (2004). The fusiform face area subserves face perception, not generic within-category identification. Nature Neuroscience, Vol. 7, No. 5, (555-562), ISSN:1097-06256.

Grood, E. S. and Suntay, W. J. (1983). A joint coordinate system for the clinical description of three-dimensional motions: Application to the knee. Journal of Biomechanical Engineering, Vol. 105, (136-144), ISSN:0148-0731.

Hale, J. G., Pollick, F. E., and Tzoneva, M. (2003). The visual categorization of humanoid movement as natural. Proceedings of the IEEE-RAS/RSJ International Conference on Humanoid Robots, ISBN:3-00-012047-5, Munich, Germany, 2003.10.

Kanda, T., Ishiguro, H., Ono, T., Imai, M., and Mase, K. (2002). Development and evaluation of an interactive robot "Robovie". Proceedings of the IEEE International Conference on Robotics and Automation, pp. 1848-1855, ISBN:0-7803-7272-7, Washington D.C., USA, 2002.05 .

Kashima, T. and Isurugi, Y. (1998).Trajectory formation based on physiological characteristics of skeletal muscles. Biological Cybernetics, Vol. 78, No. 6, (413-422), ISSN:0340-1220.

Kawato, M., Furukawa, K., and Suzuki, R. (1987). A hierarchical neural network model for control and learning of voluntary movement. Biological Cybernetics, Vol. 57, (169185), ISSN:0340-1220. 
Leardini, A., Chiari, L., Croce, U. D., and Cappozzo, A. (2005). Human movement analysis using stereophotogrammetry Part 3. Soft tissue artifact assessment and compensation. Gait and Posture, Vol. 21, (212-225), ISSN:0966-6362.

Minato, T., Shimada, M., Ishiguro, H., and Itakura, S. (2004). Development of an android robot for studying human-robot interaction. Proceedings of the 17th International Conference on Industrial \& Engineering Applications of Artificial Intelligence $\mathcal{E}$ Expert Systems, pp. 424-434, ISBN:3-540-22007-0, Ottawa, Canada, 2004.05.

Minato, T., Shimada, M., Itakura, S., Lee, K., and Ishiguro, H. (2006). Evaluating the human likeness of an android by comparing gaze behaviors elicited by the android and a person. Advanced Robotics, Vol. 20, No. 10, (1147-1163), ISSN:0169-1864.

Mori, M. (1970). Bukimi no tani [the uncanny valley] (in Japanese). Energy, Vol. 7, No. 4, (3335), ISSN:0013-7464.

Nakaoka, S., Nakazawa, A., Yokoi, K., Hirukawa, H., and Ikeuchi, K. (2003). Generating whole body motions for a biped humanoid robot from captured human dances. Proceedings of the IEEE International Conference on Robotics and Automation, ISBN:07803-7737-0, Taipei, Taiwan, 2003.09.

Okada, M., Ban, S., and Nakamura, Y. (2002). Skill of compliance with controlled charging/discharging of kinetic energy. Proceeding of the IEEE International Conference on Robotics and Automation, pp. 2455-2460, ISBN:0-7803-7272-7, Washington D.C., USA, 2002.05.

Oyama, E., Agah, A., MacDorman, K. F., Maeda, T., and Tachi, S. (2001a). A modular neural network architecture for inverse kinematics model learning. Neurocomputing, Vol. 38-40, (797-805), ISSN:0925-2312.

Oyama, E., Chong, N. Y., Agah, A., Maeda, T., Tachi, S., and MacDorman, K. F. (2001b). Learning a coordinate transformation for a human visual feedback controller based on disturbance noise and the feedback error signal. Proceedings of the IEEE International Conference on Robotics and Automation, ISBN:0-7803-6576-3, Seoul, Korea, 2001.05.

Oyama, E., MacDorman, K. F., Agah, A., Maeda, T., and Tachi, S. (2001c). Coordinate transformation learning of a hand position feedback controller with time delay. Neurocomputing, Vol. 38-40, (1503-1509), ISSN:0925-2312.

Riley, M., Ude, A., and Atkeson, C. G. (2000). Methods for motion generation and interaction with a humanoid robot: Case studies of dancing and catching. Proceedings of AAAI and CMU Workshop on Interactive Robotics and Entertainment, pp.35-42, Pennsylvania, USA, 2000.04.

Shimada, M., Minato, T., Itakura, S., and Ishiguro, H. (2006). Evaluation of android using unconscious recognition. Proceedings of the IEEE-RAS International Conference on Humanoid Robots, pp. 157-162, ISBN:1-4244-0200-X, Genova, Italy, 2006.12.

Veldpaus, F. E., Woltring, H. J., and Dortmans, L. J. M. G. (1988). A least squares algorithm for the equiform transformation from spatial marker co-ordinates. Journal of Biomechanics, Vol. 21, (45-54), ISSN:0021-9290.

Yoshikai, T., Mizuuchi, I., Sato, D., Yoshida, S., Inaba, M., and Inoue, H. (2003).

Behavior system design and implementation in spined muscle-tendon humanoid "Kenta". Journal of Robotics and Mechatronics, Vol. 15, No. 2, (143-152), ISSN:0915-3942. 


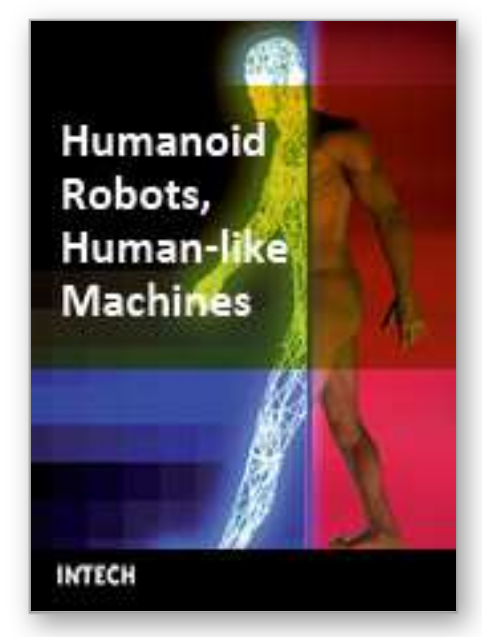

\author{
Humanoid Robots, Human-like Machines \\ Edited by Matthias Hackel
}

ISBN 978-3-902613-07-3

Hard cover, 642 pages

Publisher I-Tech Education and Publishing

Published online 01, June, 2007

Published in print edition June, 2007

In this book the variety of humanoid robotic research can be obtained. This book is divided in four parts: Hardware Development: Components and Systems, Biped Motion: Walking, Running and Self-orientation, Sensing the Environment: Acquisition, Data Processing and Control and Mind Organisation: Learning and Interaction. The first part of the book deals with remarkable hardware developments, whereby complete humanoid robotic systems are as well described as partial solutions. In the second part diverse results around the biped motion of humanoid robots are presented. The autonomous, efficient and adaptive two-legged walking is one of the main challenge in humanoid robotics. The two-legged walking will enable humanoid robots to enter our environment without rearrangement. Developments in the field of visual sensors, data acquisition, processing and control are to be observed in third part of the book. In the fourth part some "mind building" and communication technologies are presented.

\title{
How to reference
}

In order to correctly reference this scholarly work, feel free to copy and paste the following:

Daisuke Matsui, Takashi Minato, Karl F. MacDorman and Hiroshi Ishiguro (2007). Generating Natural Motion in an Android by Mapping Human Motion, Humanoid Robots, Human-like Machines, Matthias Hackel (Ed.), ISBN: 978-3-902613-07-3, InTech, Available from:

http://www.intechopen.com/books/humanoid_robots_human_like_machines/generating_natural_motion_in_an _android_by_mapping_human_motion

\section{INTECH}

open science | open minds

\author{
InTech Europe \\ University Campus STeP Ri \\ Slavka Krautzeka 83/A \\ 51000 Rijeka, Croatia \\ Phone: +385 (51) 770447 \\ Fax: +385 (51) 686166 \\ www.intechopen.com
}

\author{
InTech China \\ Unit 405, Office Block, Hotel Equatorial Shanghai \\ No.65, Yan An Road (West), Shanghai, 200040, China \\ 中国上海市延安西路65号上海国际贵都大饭店办公楼 405 单元 \\ Phone: +86-21-62489820 \\ Fax: +86-21-62489821
}


(C) 2007 The Author(s). Licensee IntechOpen. This chapter is distributed under the terms of the Creative Commons Attribution-NonCommercial-ShareAlike-3.0 License, which permits use, distribution and reproduction for non-commercial purposes, provided the original is properly cited and derivative works building on this content are distributed under the same license. 